This is the final peer-reviewed accepted manuscript of:

Mentrelli, A. Modelling of the convective plasma dynamics in the Sun: anelastic and Boussinesq MHD systems. Ricerche mat 68, 421-433 (2019).

The final published version is available online at : https://doi.org/10.1007/s11587018-0416-6

Rights / License:

The terms and conditions for the reuse of this version of the manuscript are specified in the publishing policy. For all terms of use and more information see the publisher's website. 


\title{
Modelling of the Convective Plasma Dynamics in the Sun: Anelastic and Boussinesq MHD Systems
}

\author{
Andrea Mentrelli
}

Received: date / Accepted: date

\section{Dedicated to Tommaso Ruggeri, with true esteem and affection}

\begin{abstract}
This work deals with the mathematical modelling and asymptotic analysis of the plasma convective dynamics in the center of the Sun. The heat produced via thermonuclear fusion in the interior of the Sun is transported towards the surface first via radiation, and finally via convection. Convection is thought to be responsible for the generation of magnetic fields and is hence a very important phenomenon to be understood in detail in order to get more insight in the internal structure of the Sun. Anelastic and Boussinesq models are formally derived here from the underlying compressible MHD models and we shall prepare the ground for our future numerical works, based on Asymptotic-Preserving techniques.
\end{abstract}

Keywords Plasma modelling · Convection in the Sun · Singularly perturbed problems · compressible MHD-system · Anelastic equation · Boussinesq equation · Asymptotic-Preserving scheme

\section{Introduction}

The goal of this work is to present a hierarchy of mathematical models, starting from the compressible MHD system, in the aim to describe adequately the conductive plasma dynamics in the center of giant planets and stars, like our Sun. A large part of astrophysical research treats with the study of a ionized

Andrea Mentrelli

Department of Mathematics \& $\mathrm{AM}^{2}$, University of Bologna

via Saragozza, 8

I - 40123 Bologna

E-mail: andrea.mentrelli@unibo.it 
gas (plasma) under the influence of gravitational and magnetic forces. Our particular aim here is to investigate the process of (turbulent) heat transfer by motion.

To be more precise, let us start by describing the physical context. The Sun is a "dwarf" star of average size, temperature and brightness, which is held together by its own gravity, in other words it is a self-gravitating sphere of plasma. The interior of the Sun is divided into three regions, defined by the different processes that occur there (see Figure 1). First, there is the core, where the thermonuclear fusion reactions take place, transforming hydrogen nuclei into helium nuclei. These reactions release the energy that escapes ultimately from the solar surface as visible light. On its way towards the Sun's surface, this energy is first transported by radiation (photons) through the radiative zone, a phenomenon which lasts about a million of years, due to the high density of the Sun's interior. As the temperature gets lower, the radiation becomes less powerful and another process is set into motion in order to transport more efficiently the energy, namely the convection process. It is thought that most of the solar magnetic activity is driven by turbulent flows, rotation and shear in this convective zone and the origin of the magnetic field is believed to be in the tachocline, which is the thin interface separating the radiative zone from the convective one. Anyhow, the dynamics of the solar interior is up to now still poorly understood. Given the wide range of temporal and spacial scales coexisting in the physical processes arising in the Sun, it is a great challenge to model self-consistently the solar interior and the here occurring dynamo effects. All these phenomena require state-of-the-art numerical schemes supported by rigorous mathematical results.
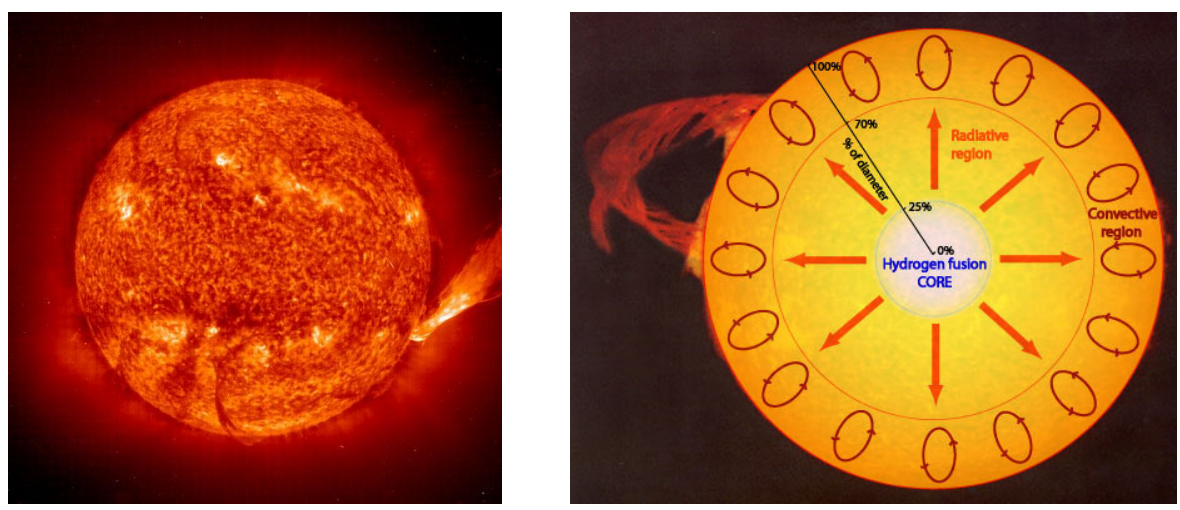

Fig. 1 Left: Turbulences in the Sun; Right: Different regions of the Sun (Core; radiative and convective region, separated by the tachocline).

Our aim in this work is the mathematical modelling and analysis of the plasma dynamics in the solar convective region. The compressible MHD equations, adapted for a precise description of this convective process [15], are the 
following:

$$
(M H D)\left\{\begin{array}{l}
\partial_{t} \rho+\nabla \cdot(\rho \mathbf{u})=0, \\
\rho\left[\partial_{t} \mathbf{u}+(\mathbf{u} \cdot \nabla) \mathbf{u}\right]=-\nabla p+\rho g+2 \rho(\mathbf{u} \times \Omega) \\
\quad+\frac{1}{4 \pi}(\nabla \times \mathbf{B}) \times \mathbf{B}+\nabla \cdot \mathcal{D}, \\
\rho T\left[\partial_{t} S+(\mathbf{u} \cdot \nabla) S\right]=\nabla \cdot\left(\kappa_{T} c_{p} \rho \nabla T\right)+\nabla \cdot\left(\kappa_{S} \rho T \nabla S\right) \\
\quad+\frac{\eta}{4 \pi}(\nabla \times \mathbf{B})^{2}, \\
\partial_{t} \mathbf{B}=\nabla \times(\mathbf{u} \times \mathbf{B})-\nabla \times(\eta \nabla \times \mathbf{B}), \\
\nabla \cdot \mathbf{B}=0 .
\end{array}\right.
$$

The plasma gas flow in the interior of the Sun is described in terms of the mass density $\rho(t, \mathbf{x})$, its mean velocity $\mathbf{u}(t, \mathbf{x})$, the temperature $T(t, \mathbf{x})$, the pressure $p(t, \mathbf{x})$, the specific entropy $S(t, \mathbf{x})$ and the self-consistent magnetic field B. Let us also mention that gravity acts radially inwards. Furthermore, $\Omega$ is the angular velocity of the rotating Sun, the term $2 \rho(\mathbf{u} \times \Omega)$ being the Coriolis force term, $\mathcal{D}$ the viscous stress tensor and $\eta$ the magnetic viscosity. In most astrophysical applications, the turbulent thermal diffusivity (or eddy diffusivity) dominates the standard thermal diffusivity (or molecular diffusivity). Due to this considerations, we introduce apart from the thermal diffusivity term $\nabla \cdot\left(\kappa_{T} c_{p} \rho \nabla T\right)$ an additional, more appropriate, entropy diffusion term $\nabla \cdot\left(\kappa_{S} \rho T \nabla S\right)$, as used in the "mixing length theory" [16], and suppose that the turbulent and thermal diffusion coefficients, respectively $\kappa_{T}$ and $\kappa_{S}$, are such that $\kappa_{T} \ll \kappa_{S}$.

System (1) is finally supplied, for its closure, with the following equations of state (ideal gas assumptions), relating the thermonuclear variables

$$
p=\mathcal{R} \rho T=\frac{\gamma-1}{\gamma} c_{p} \rho T, \quad S=\frac{c_{p}}{\gamma}\left(\ln \frac{p}{p_{\diamond}}-\gamma \ln \frac{\rho}{\rho_{\diamond}}\right)=c_{v} \ln \left(\frac{p}{p_{\diamond}} \frac{\rho_{\diamond}^{\gamma}}{\rho^{\gamma}}\right),
$$

being $p_{\diamond}$ and $\rho_{\diamond}$ arbitrary values for the pressure and density, respectively; $\mathcal{R}:=c_{p}-c_{v}$ the universal gas constant, and $\gamma=c_{p} / c_{v}$ the adiabatic constant $(\gamma=5 / 3$ for monoatomic gases and $\gamma=7 / 3$ for diamtomic molecular gases), where $c_{p}$ and $c_{v}$ are the specific heat capacities at constant pressure and constant volume, respectively, which we shall assume to be constant.

The difficulty in the numerical resolution of the fully compressible MHD system (1) is that one has to resolve the very rapid sound wave dynamics, fact which very rapidly becomes a computationally expensive task. Reduced, approximate models, such as the Anelastic, Boussinesq or simply incompressible models are then introduced, to filter out the sound waves and to permit the choice of coarser grids. To derive these approximate models, one has first to identify the physical regime of interest, introduce reference values as well as some adimensional parameters (Mach and Froude numbers, for instance) and perform finally the adequate asymptotic limits. 
Starting from the MHD system (1) (with $\mathbf{B} \equiv 0$ ) and performing the adequate scaling corresponding to the convective plasma dynamics, we shall in this work obtain the following singularly perturbed problem

$$
(P)^{\epsilon, \alpha}\left\{\begin{array}{l}
\partial_{t} \rho+\nabla \cdot(\rho \mathbf{u})=0 \\
\rho\left[\partial_{t} \mathbf{u}+(\mathbf{u} \cdot \nabla) \mathbf{u}\right]=-\frac{1}{\epsilon^{2}} \nabla p+\frac{\alpha}{\epsilon^{2}} \rho g \\
\rho T\left[\partial_{t} S+(\mathbf{u} \cdot \nabla) S\right]=\epsilon^{2} \nabla \cdot(\rho \nabla T)+\nabla \cdot(\rho T \nabla S),
\end{array}\right.
$$

system which will be the starting model for obtaining several different reduced models. Not all of them are well suited for the solar convection region, however for completeness we shall mention them all.

Depending on the magnitude of both occurring parameters $\alpha$ and $\epsilon$, mainly three models are obtained. In the case $\alpha=\mathcal{O}(1)$ and $\epsilon \rightarrow 0$, we shall get in the limit the so-called anelastic model. In the case $\alpha=\mathcal{O}(\epsilon)$ and $\epsilon \rightarrow 0$, one obtains the well-known Boussinesq model (used to describe ocean circulation or katabatic winds), whereas $\alpha=\mathcal{O}\left(\epsilon^{2}\right)$ and $\epsilon \rightarrow 0$ lead to the well-known incompressible equations (low-Mach limit). In Section 2 we present the physical scaling leading to the dimensionless form of the problem (3). In Section 3 we perform the formal asymptotic limits permitting to get the different reduced models and we shall also single out the model most suited for the convection zone dynamics. Finally the aim of Section 4 is to put the basis for our future numerical works. Indeed, our final aim is to design an efficient numerical scheme, based on asymptotic-preserving techniques, which will be able to solve $(P)^{\epsilon, \alpha}$ uniformly accurate and stable in $\epsilon$ (and $\alpha$ ), without having to adapt the grid to the perturbation parameters $\epsilon$ and $\alpha$. Such an AP-scheme could be very useful for numerical simulations of the convective solar dynamics, as it would permit coarser grids and hence a considerable gain in simulation time.

\section{Physical scaling}

To focus only on some specific multiscale mathematical difficulties in problem (1), we shall assume in the following that $\mathbf{B} \equiv 0, \Omega \equiv 0$ and $\mathcal{D} \equiv 0$. Some of these assumptions are not completely unphysical, as one can assume for example that the centrifugal force is small compared to the gravitational force. Moreover, in astrophysical fluid dynamics the presence of solid walls and viscosity is often unimportant [20], allowing to safely neglect the viscous stress tensor $\mathcal{D}$. However, the magnetic field is important in the convection zone, such that in a further work, one should take into account for its influence. In this first step however, we shall neglect $\mathbf{B}$, and characterize the regime we are interested in by the following scales $[8,16,21]$ :

- Diffusion coefficients: We introduce a first small parameter $\tau$, measuring the ratio between the thermal and the turbulent diffusivities, i.e.

$$
\frac{\kappa_{S}}{\kappa_{T}}=\frac{1}{\tau^{2}}
$$


- Length, Time and Velocity scales: Let us denote by $\hat{x}$ the length-scale of reference, being the scale of the considered convective motion; the reference time $\hat{t}$ is chosen to be the entropic diffusion time $\hat{t}=\hat{x}^{2} / \kappa_{S}$, with $\kappa_{S}$ the turbulent diffusion coefficient. Then the reference velocity is given by

$$
\hat{u}=\frac{\hat{x}}{\hat{t}}=\frac{\kappa_{S}}{\hat{x}} .
$$

- Sound waves/Gravity waves: We shall assume that the convection velocity $\hat{u}$ is much smaller that the sound speed $(c=\sqrt{\hat{p} / \hat{\rho}})$ as well as the gravity fall speed $\left(u_{G F}=\sqrt{\hat{\rho} \hat{x}}\right)$. This permits to introduce the Mach and the Froude numbers

$$
\frac{\hat{p}}{\hat{\rho} \hat{u}^{2}}=\frac{1}{M a^{2}}, \quad \frac{\hat{g} \hat{t}}{\hat{u}}=\frac{\hat{g} \hat{x}}{\hat{u}^{2}}=\frac{1}{F r^{2}}
$$

- Stratification: The degree of stratification of our model is defined by a parameter $\alpha$, called Boussinesq number and defined as

$$
\alpha:=\frac{M a^{2}}{F r^{2}}=\frac{\hat{x} \hat{\rho} \hat{g}}{\hat{p}}=\frac{\hat{x}}{\hat{d}}, \quad \hat{d}:=\frac{\hat{p}}{\hat{\rho} \hat{g}}
$$

where $\hat{d}$ represents nothing else than the pressure scale height of the fluid. Letting $\alpha$ tend to zero means letting the ratio between the typical domain scale-length and the pressure scale tend to zero, which means removing the height limitation and leading thus to the Boussinesq approximation.

- Entropy: The entropy $S$ is scaled by the specific heat capacity at constant pressure. We shall also assume that $S$ is fixed at the inner boundary $r=r_{i}$ and the outer boundary $r=r_{o}$ of the convection shell (represented in Figure 2), such that the entropy gradient instability criterion (in the radial direction) $\partial S / \partial r<0$ is satisfied. Finally we shall introduce the essential parameter $\epsilon$, comparing the typical length-scale $\hat{x}$ with the entropy-gradient length-scale. Briefly, one has

$$
\hat{S}=c_{p}, \quad S_{i}=\hat{S} \Delta S>0, \quad S_{o}=0, \quad \hat{x} \frac{\hat{\nabla} S}{\hat{S}}=\Delta S=\epsilon^{2} .
$$

- Thermodynamic variables: The state equations are also made dimensionless, by imposing the relations

$$
\hat{p}=\mathcal{R} \hat{\rho} \hat{T}, \quad \hat{S}=\frac{c_{p}}{\gamma}\left(\ln \frac{\hat{p}}{p_{\diamond}}-\gamma \ln \frac{\hat{\rho}}{\rho_{\diamond}}\right)=c_{v} \ln \left(\frac{\hat{p}}{p_{\diamond}} \frac{\rho_{\diamond}^{\gamma}}{\hat{\rho}^{\gamma}}\right) .
$$

Having defined all the characteristic values for our problem, let us now decompose each quantity in its reference value and an adimensional function, as 
$\rho=\hat{\rho} \rho_{*}$, to get the system

$$
\left\{\begin{array}{l}
\partial_{t_{*}} \rho_{*}+\frac{\hat{t} \hat{u}}{\hat{x}} \nabla_{*} \cdot\left(\rho_{*} \mathbf{u}_{*}\right)=0 \\
\rho_{*}\left[\partial_{t_{*}} \mathbf{u}_{*}+\frac{\hat{t} \hat{u}}{\hat{x}}\left(\mathbf{u}_{*} \cdot \nabla_{*}\right) \mathbf{u}_{*}\right]=-\frac{\hat{p}}{\hat{\rho} \hat{u}^{2}} \nabla_{*} p_{*}+\frac{\hat{t} \hat{g}}{\hat{u}} \rho_{*} g_{*} \\
\rho_{*} T_{*}\left[\partial_{t_{*}} S_{*}+\frac{\hat{t} \hat{u}}{\hat{x}}\left(\mathbf{u}_{*} \cdot \nabla_{*}\right) S_{*}\right] \\
\quad=\nabla_{*} \cdot\left(\frac{\kappa_{T}}{\kappa_{S}} \rho_{*} \nabla_{*} T_{*}\right)+\frac{\hat{t} \hat{u}}{\hat{x}} \nabla_{*} \cdot\left(\rho_{*} T_{*} \nabla_{*} S_{*}\right) .
\end{array}\right.
$$

For the description of the solar convective motion, we are interested in low Mach regimes and shall assume the following ordering

$$
M a=\epsilon, \quad F r=\epsilon / \sqrt{\alpha}, \quad \tau=\epsilon,
$$

which embodies for simplicity only one perturbation parameter $\epsilon \ll 1$, where $\alpha(\epsilon)$ shall be $\epsilon$-dependent (of order $\mathcal{O}(1), \mathcal{O}(\sqrt{\epsilon})$ or $\mathcal{O}(\epsilon)$ ), leading to different regimes.

Taking into account for all the above mentioned scaling considerations, and omitting all the stars, we obtain the following singularly-perturbed system for the unknowns $\left(\rho^{\epsilon, \alpha}, \mathbf{u}^{\epsilon, \alpha}, S^{\epsilon, \alpha}\right)$

$$
(P)^{\epsilon, \alpha}\left\{\begin{array}{l}
\partial_{t} \rho^{\epsilon, \alpha}+\nabla \cdot\left(\rho^{\epsilon, \alpha} \mathbf{u}^{\epsilon, \alpha}\right)=0 \\
\rho^{\epsilon, \alpha}\left[\partial_{t} \mathbf{u}^{\epsilon, \alpha}+\left(\mathbf{u}^{\epsilon, \alpha} \cdot \nabla\right) \mathbf{u}^{\epsilon, \alpha}\right]=-\frac{1}{\epsilon^{2}} \nabla p^{\epsilon, \alpha}+\frac{\alpha}{\epsilon^{2}} \rho^{\epsilon, \alpha} g \\
\rho^{\epsilon, \alpha} T^{\epsilon, \alpha}\left[\partial_{t} S^{\epsilon, \alpha}+\left(\mathbf{u}^{\epsilon, \alpha} \cdot \nabla\right) S^{\epsilon, \alpha}\right]=\epsilon^{2} \nabla \cdot\left(\rho^{\epsilon, \alpha} \nabla T^{\epsilon, \alpha}\right) \\
+\nabla \cdot\left(\rho^{\epsilon, \alpha} T^{\epsilon, \alpha} \nabla S^{\epsilon, \alpha}\right),
\end{array}\right.
$$

associated with the rescaled equations of state

$$
p^{\epsilon, \alpha}=\rho^{\epsilon, \alpha} T^{\epsilon, \alpha}, \quad S^{\epsilon, \alpha}=\frac{1}{\gamma} \ln \left(\frac{p^{\epsilon, \alpha}}{\left(\rho^{\epsilon, \alpha}\right)^{\gamma}}\right)+1,
$$

as well as initial and boundary conditions. The initial conditions for $\rho^{\epsilon, \alpha}, \mathbf{u}^{\epsilon, \alpha}$, $S^{\epsilon, \alpha}$ will be specified in each particular case and will be slight perturbations of a hydrostatic state (see Eq. (11) for example).

Concerning the boundary conditions, let us mention that for simplicity reasons, the considered simulation domain $\Omega$ will be chosen to be a rectangle $\left[0, L_{x}\right] \times\left[0, L_{y}\right] \times\left[0, L_{z}\right]$ with the gravity acting vertically in the direction $z$, i.e. $g=(0,0, g(z))$. This rectangle is a simplified description of the spherical convection shell, with the outer boundary in $z=0$ and the inner one in $z=L_{z}$ (see Fig. 2). Thus, all the quantities are supposed to be periodic with respect to $(x, y)$. Moreover, stress-free impenetrable conditions on the velocity are assumed, i.e.

$$
u_{z}^{\epsilon, \alpha}=\partial_{z} u_{x}^{\epsilon, \alpha}=\partial_{z} u_{y}^{\epsilon, \alpha}=0 \quad \text { for } \quad z=0, \quad z=L_{z} .
$$


The entropy is supposed to be known on the upper (interior part of the shell) and lower (outer part) boundary, with $S_{o}^{\epsilon, \alpha}=0$ for $z=0$ and $S_{i}^{\epsilon, \alpha}=\Delta S=\epsilon^{2}$ for $z=L_{z}$. For the density, we suppose $\rho^{\epsilon, \alpha}$ to be known and constant on the inner and outer part of the shell, namely $\rho^{\epsilon, \alpha}(t, x, y, 0)=\rho_{o}$ and $\rho^{\epsilon, \alpha}\left(t, x, y, L_{z}\right)=\rho_{i}$.

Model (6)-(7) is our starting point for the derivation of a hierarchy of reduced models, permitting to describe different regimes of low Mach motions, especially the one we are interested in, namely the convective plasma motion in the Sun.

\section{Asymptotic limits}

Starting from the singularly-perturbed problem (6)-(7), let us perform in this section the anelastic, the Boussinesq as well as the incompressible asymptotics, letting the parameter $\epsilon$ tend towards zero. This procedure shall be based on formal asymptotic techniques, such as the Hilbert expansion. The interested reader can find some more rigorous asymptotic studies in $[6,7]$.

3.1 The anelastic approximation: $\alpha=\mathcal{O}(1), \epsilon \ll 1$.

The anelastic regime corresponds to the scaling $M a^{2}=\epsilon^{2}, F r^{2}=\epsilon^{2}$ and a Boussinesq number of $\alpha:=M a^{2} / F r^{2}=1$. The starting model is thus

$$
(P)_{A}^{\epsilon}\left\{\begin{array}{l}
\partial_{t} \rho^{\epsilon}+\nabla \cdot\left(\rho^{\epsilon} \mathbf{u}^{\epsilon}\right)=0 \\
\rho^{\epsilon}\left[\partial_{t} \mathbf{u}^{\epsilon}+\left(\mathbf{u}^{\epsilon} \cdot \nabla\right) \mathbf{u}^{\epsilon}\right]=-\frac{1}{\epsilon^{2}} \nabla p^{\epsilon}+\frac{1}{\epsilon^{2}} \rho^{\epsilon} g \\
\rho^{\epsilon} T^{\epsilon}\left[\partial_{t} S^{\epsilon}+\left(\mathbf{u}^{\epsilon} \cdot \nabla\right) S^{\epsilon}\right]=\epsilon^{2} \nabla \cdot\left(\rho^{\epsilon} \nabla T^{\epsilon}\right)+\nabla \cdot\left(\rho^{\epsilon} T^{\epsilon} \nabla S^{\epsilon}\right),
\end{array}\right.
$$

completed with the equations of state (7), initial and boundary conditions. For simplicity, we shall omit in the following the $\epsilon$-dependencies of the functions. To obtain the limit model of (8) as $\epsilon \rightarrow 0$, we shall suppose that all quantities can be expanded in a power series in the small parameter $\epsilon$ (Hilbert expansion)

$$
\left\{\begin{array}{l}
\rho(t, \mathbf{x})=\bar{\rho}(t, \mathbf{x})+\epsilon^{2} \rho^{\prime}(t, \mathbf{x})+\cdots, \quad \mathbf{u}(t, \mathbf{x})=\overline{\mathbf{u}}(t, \mathbf{x})+\epsilon^{2} \mathbf{u}^{\prime}(t, \mathbf{x})+\ldots \\
p(t, \mathbf{x})=\bar{p}(t, \mathbf{x})+\epsilon^{2} p^{\prime}(t, \mathbf{x})+\ldots, \\
T(t, \mathbf{x})=\bar{T}(t, \mathbf{x})+\epsilon^{2} T^{\prime}(t, \mathbf{x})+\ldots, \quad S(t, \mathbf{x})=\bar{S}(t, \mathbf{x})+\epsilon^{2} S^{\prime}(t, \mathbf{x})+\ldots
\end{array}\right.
$$

Inserting this Ansatz in the original problem (8) and comparing the terms of the same order in $\epsilon$ yields a hierarchy of equations to be solved to get some information about the distinct terms in the Hilbert expansion; in particular 
we are interested in computing only the macroscopic quantities. The first constraint tells us that for $\epsilon \rightarrow 0$, the limit functions, denoted here by a bar, shall satisfy the hydrostatic equilibrium equation

$$
\nabla \bar{p}=\bar{\rho} g, \quad \text { where } \quad g=(0,0, g(z)) .
$$

This implies immediately that in the limit of vanishing $\epsilon$, the pressure and the density are functions depending only on the vertical variable, i.e. $\bar{p}=\bar{p}(t, z)$ and $\bar{\rho}=\bar{\rho}(t, z)$. The equations of state (7) yield then the information that the temperature, as well as the entropy, are in the limit $\epsilon \rightarrow 0$ also of the form $\bar{T}=\bar{T}(t, z)$ and $\bar{S}=\bar{S}(t, z)$, respectively.

Now the boundary conditions for $\rho$ and $S$, which are time-independent on the inner and outer part of our shell, permit immediately to show (with the help of the equations of state) that all these quantities are also timeindependent. Finally, the scaling conditions for the entropy (4) yield that $\bar{S}(z) \equiv$ const $=0$. Altogether one gets the dependencies

$$
\bar{\rho}(z), \quad \bar{T}(z), \quad \bar{p}(z), \quad \bar{S} \equiv \text { const }=0 .
$$

Nothing is known for the moment about the velocity $\bar{u}(t, \mathbf{x})$. In order to obtain the limit model for $\epsilon \rightarrow 0$, we go further and match in (8) the terms of order zero in $\epsilon^{2}$. This yields the anelastic model

$$
(P)_{A}^{0}\left\{\begin{array}{l}
\nabla \cdot(\bar{\rho} \overline{\mathbf{u}})=0 \\
\partial_{z} \bar{p}=\bar{\rho} g \\
\bar{\rho}\left[\partial_{t} \overline{\mathbf{u}}+(\overline{\mathbf{u}} \cdot \nabla) \overline{\mathbf{u}}\right]=-\nabla p^{\prime}+\rho^{\prime} g \\
\bar{\rho} \bar{T}\left[\partial_{t} S^{\prime}+(\overline{\mathbf{u}} \cdot \nabla) S^{\prime}\right]=\partial_{z}\left[\bar{\rho} \partial_{z} \bar{T}\right]+\nabla \cdot\left[\bar{\rho} \bar{T} \nabla S^{\prime}\right]
\end{array}\right.
$$

If one supplies now the starting model (8) with "well-prepared" initial conditions, meaning perturbations of a hydrostatic equilibrium, i.e.

$$
\rho(0, \mathbf{x})=\bar{\rho}(z)+\epsilon^{2} \rho_{i n}^{\prime}(\mathbf{x}), \quad \mathbf{u}(0, \mathbf{x})=\mathbf{u}_{i n}(\mathbf{x}), \quad S(0, \mathbf{x})=\epsilon^{2} S_{i n}^{\prime}(\mathbf{x})
$$

the anelastic system (10), associated with the equations of state (7), which at leading order read

$$
\bar{p}=\bar{\rho} \bar{T}, \quad \frac{1}{\gamma} \ln (\bar{p})-\ln (\bar{\rho})+1=0, \quad \frac{1}{\gamma} \frac{p^{\prime}}{\bar{p}}-\frac{\rho^{\prime}}{\bar{\rho}}=S^{\prime},
$$

is a well-posed model, permitting to compute the remaining quantities $\bar{p}, \bar{T}$, $\overline{\mathbf{u}}, p^{\prime}, \rho^{\prime}$, and $S^{\prime}$. 
3.2 The Boussinesq approximation: $\alpha=\mathcal{O}(\epsilon), \epsilon \ll 1$.

The Boussinesq regime corresponds to the scaling $M a^{2}=\epsilon^{2}, F r^{2}=\epsilon$ and a Boussinesq number of $\alpha:=M a^{2} / F r^{2}=\epsilon$. The starting model is hence

$$
(P)_{B}^{\epsilon}\left\{\begin{array}{l}
\partial_{t} \rho+\nabla \cdot(\rho \mathbf{u})=0 \\
\rho\left[\partial_{t} \mathbf{u}+(\mathbf{u} \cdot \nabla) \mathbf{u}\right]=-\frac{1}{\epsilon^{2}} \nabla p+\frac{1}{\epsilon} \rho g \\
\rho T\left[\partial_{t} S+(\mathbf{u} \cdot \nabla) S\right]=\epsilon^{2} \nabla \cdot(\rho \nabla T)+\nabla \cdot(\rho T \nabla S) .
\end{array}\right.
$$

Supposing now that each quantity has a Hilbert expansion of the form

$$
\left\{\begin{array}{l}
\rho(t, \mathbf{x})=\bar{\rho}(t, \mathbf{x})+\epsilon \rho^{\prime}(t, \mathbf{x})+\cdots, \quad \mathbf{u}(t, \mathbf{x})=\overline{\mathbf{u}}(t, \mathbf{x})+\epsilon \mathbf{u}^{\prime}(t, \mathbf{x})+\cdots \\
p(t, \mathbf{x})=\bar{p}(t, \mathbf{x})+\epsilon p_{1}^{\prime}(z)+\epsilon^{2} p_{2}^{\prime}(t, \mathbf{x})+\cdots, \\
T(t, \mathbf{x})=\bar{T}(t, \mathbf{x})+\epsilon T^{\prime}(t, \mathbf{x})+\cdots, \quad S(t, \mathbf{x})=\bar{S}(t, \mathbf{x})+\epsilon S^{\prime}(t, \mathbf{x})+\cdots
\end{array}\right.
$$

inserting then this Ansatz in (12) and comparing the terms of the same order in $\epsilon$, yields firstly the two constraints

$$
\nabla \bar{p}=0, \quad \nabla p_{1}^{\prime}=\bar{\rho} g, \quad g=(0,0, g(z)) .
$$

The first constraint implies immediately that in the limit $\epsilon \rightarrow 0$ the pressure is a function only of the time, i.e. $\bar{p}(t)$. The second constraint gives the dependences $\bar{\rho}(t, z), p_{1}^{\prime}(t, z)$ and with the help of the equations of state (7) also $\bar{T}(t, z)$ and $\bar{S}(t, z)$. Going forward, the boundary conditions for $\rho$, imply that $\bar{\rho}$ is independent on the time and the scaling conditions for the entropy (4) yield that $\bar{S}(t, z) \equiv$ const $=0$, such that finally we obtain for the limit quantities, the dependencies

$$
\bar{\rho} \equiv \text { const }, \quad \bar{T} \equiv \text { const }, \quad \bar{S} \equiv \text { const }=0, \quad p_{1}^{\prime}(z), \quad \bar{p} \equiv \text { const } .
$$

In order to obtain the limit model for $\epsilon \rightarrow 0$, we go one step further in the identification of the power terms in $\epsilon$, and obtain the Boussinesq system

$$
(P)_{B}^{0}\left\{\begin{array}{l}
\nabla \cdot \overline{\mathbf{u}}=0 \\
\partial_{z} p_{1}^{\prime}(z)=\bar{\rho} g \\
\bar{\rho}\left[\partial_{t} \overline{\mathbf{u}}+(\overline{\mathbf{u}} \cdot \nabla) \overline{\mathbf{u}}\right]=-\nabla p_{2}^{\prime}+\rho^{\prime} g \\
\partial_{t} S^{\prime}+(\overline{\mathbf{u}} \cdot \nabla) S^{\prime}-\Delta S^{\prime}=0 .
\end{array}\right.
$$

Again, supplying this last system with "well-prepared" initial conditions of the type

$$
\rho(0, \mathbf{x})=\bar{\rho}+\epsilon \rho_{i n}^{\prime}(\mathbf{x}), \quad \mathbf{u}(0, \mathbf{x})=\mathbf{u}_{i n}(\mathbf{x}), \quad S(0, \mathbf{x})=\epsilon S_{i n}^{\prime}(\mathbf{x}),
$$

shall permit, together with $(7)$, which at leading order write

$$
\bar{p}=\bar{\rho} \bar{T}, \quad \frac{1}{\gamma} \ln (\bar{p})-\ln (\bar{\rho})+1=0, \quad \frac{1}{\gamma} \frac{p_{1}^{\prime}}{\bar{p}}-\frac{\rho^{\prime}}{\bar{\rho}}=S^{\prime},
$$

to compute the remaining quantities $\bar{p}, \bar{T}, \overline{\mathbf{u}}, p_{1}^{\prime}, p_{2}^{\prime}, \rho^{\prime}, S^{\prime}$. 
3.3 The low Mach number approximation: $\alpha=\mathcal{O}\left(\epsilon^{2}\right), \epsilon \ll 1$.

The low Mach number regime has been widely investigated in literature $[1,5$, 11], we mention it here only for completeness. It corresponds to the scaling $M a^{2}=\epsilon^{2}, F r^{2}=1$ and a Boussinesq number of $\alpha:=M a^{2} / F r^{2}=\epsilon^{2}$. The starting model is

$$
(P)_{M}^{\epsilon}\left\{\begin{array}{l}
\partial_{t} \rho+\nabla \cdot(\rho \mathbf{u})=0 \\
\rho\left[\partial_{t} \mathbf{u}+(\mathbf{u} \cdot \nabla) \mathbf{u}\right]=-\frac{1}{\epsilon^{2}} \nabla p+\rho g \\
\rho T\left[\partial_{t} S+(\mathbf{u} \cdot \nabla) S\right]=\epsilon^{2} \nabla \cdot(\rho \nabla T)+\nabla \cdot(\rho T \nabla S) .
\end{array}\right.
$$

In the limit $\epsilon \rightarrow 0$, one gets, following the same procedure as presented above, the incompressible equations

$$
(P)_{M}^{0}\left\{\begin{array}{l}
\nabla \cdot \overline{\mathbf{u}}=0 \\
\nabla \bar{p}=0 \\
\bar{\rho}\left[\partial_{t} \overline{\mathbf{u}}+(\overline{\mathbf{u}} \cdot \nabla) \overline{\mathbf{u}}\right]=-\nabla p^{\prime}+\bar{\rho} g \\
\partial_{t} S^{\prime}+(\overline{\mathbf{u}} \cdot \nabla) S^{\prime}-\Delta S^{\prime}=0,
\end{array}\right.
$$

completed with "well-prepared" initial conditions (15), boundary conditions as well as the equations of state (7), yielding the unknowns $\overline{\mathbf{u}}, p^{\prime}, S^{\prime}$.

\subsection{Choice of the solar convective model.}

Not all of the above mentioned reduced models are well suited to describe the convective dynamics of the stellar plasma. The most precise one is clearly the starting MHD-system (1) - in our simplified framework this corresponds to system (6). The Boussinesq model (14) is not really adequate for this convective motion, as it does not take into account for the fact that the density is much larger in the deep solar interior than in its upper regions. Neither does the low Mach-number approximation (17). The Anelastic model (10) can be considered as intermediate between the MHD-model on one hand and the Boussinesq model on the other hand. In contrast to the Boussinesq model, the density and all other quantities are not required to be constant in the Anelastic model, since they may vary with depth $z$. Thus the Anelastic model is incorporating some stratification, when compared to the Boussinesq model, however without having to resort to the full compressibility of the MHD-model.

In conclusion the Anelastic model (10) seems to be the most suitable approximate model for a simulation of the convective plasma dynamics in the stellar interior. It has significant numerical advantages, suppressing the acoustic waves and permitting hence larger time steps than would be necessary for the fully compressible MHD-model, and furthermore it includes more physics than the Boussinesq model. 
However, the legitimacy of the Anelastic model (10) as a reasonably accurate approximation of the MHD model (1) is only justified far from the outer layers of the solar interior. Indeed, close to the surface (photosphere) the velocities of the plasma gas start to become larger and larger, exceeding even the sound speed near the surface. In these regions it is hence more accurate to resort to the more precise MHD-system, or in our simplified situation to the starting model (8) with $\epsilon \sim 1$. Consequently the appropriate description of this convective region is the singularly-perturbed system (8), with $\epsilon$ running through all values between $[0,1]$, in particular $\epsilon \ll 1$ in the interior region and $\epsilon \sim 1$ in the outer region. This model shall be hence our starting point for the numerical simulations we plan to perform in a future paper.

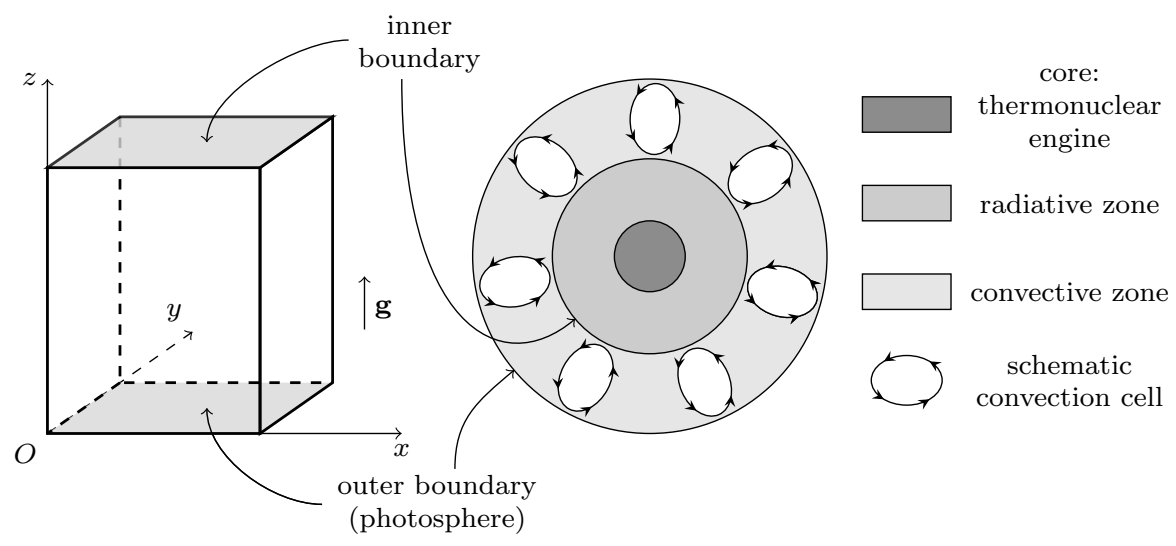

Fig. 2 Schematic representation of the simulation domain and of the stellar interior zones.

\section{Numerical difficulties and basis of the forthcoming work}

Once the mathematical model for a precise description of the plasma convective dynamics in the Sun has been identified, namely the MHD-system in the Anelastic scaling (8), the issue is now to find an adequate numerical scheme for an efficient and accurate resolution, in particular a scheme able to take into account for all values of $\epsilon \in[0,1]$, leading in the limit $\epsilon \rightarrow 0$ towards a discretization of the Anelastic system (10).

Problem (8) is a typical multiscale or singularly-perturbed problem, the parameter $\epsilon$ introducing various scales in the problem. The mathematical as well as numerical studies of such problems is rather challenging, difficulties arise due to a change in type of the equation as $\epsilon \rightarrow 0$. As a consequence, the use of standard numerical schemes for a resolution of $(P)_{A}^{\epsilon}$ would require very restrictive time and/or space discretization steps of the type $\Delta t, \Delta x \sim$ 
$\mathcal{O}\left(\epsilon^{2}\right)$. This becomes rapidly too costly from a numerical point of view and consequently a numerical asymptotic study and even numerical simulations for small $\epsilon$-values, are out of reach. Moreover, standard implicit schemes (even if computationally heavy) may be uniformly stable for $0<\epsilon<1$, but yet provide a wrong solution in the limit $\epsilon \rightarrow 0$, which means the scheme is not consistent with the limit problem $(P)_{A}^{0}$. Thus the design of robust numerical methods, whose accuracy and stability do not depend on the parameter $\epsilon$, allowing even to capture the limit $\epsilon \rightarrow 0$, becomes an important task.

One approach in astrophysical simulations to tackle such problems, is to use hybrid techniques, solving $(P)_{A}^{\epsilon}$ there where $\epsilon \sim \mathcal{O}(1)$ and $(P)_{A}^{0}$ where $\epsilon$ is rather small $[9,10,15]$. Several difficulties can be encountered with this approach, for example how to locate the interface between $(P)_{A}^{\epsilon}$ and $(P)_{A}^{0}$ and what type of interface conditions to use. Thus, this approach can be difficult to implement in practice. That is the reason why several large-scale astrophysical simulations typically neglect the upper part, namely $5-10 \%$ of the solar convection zone [19]. We propose on the contrary to base our numerical method on Asymptotic-Preserving techniques, which we have extensively adopted in recent years to successfully investigate other singularly perturbed problems $[2-4,17,18]$.

Asymptotic-Preserving schemes were introduced the first time by S. Jin $[12,13]$ with the aim to cope with such singularly perturbed problems in the framework of kinetic models in a diffusive regime. The construction of these AP-schemes necessitates the existence of a well-posed limit problem $(P)_{A}^{0}$, which has to be identified beforehand. The main feature of these schemes is that they permit a precise, $\epsilon$-independent, resolution of the problem $(P)_{A}^{\epsilon}$ as well as of its limit problem $(P)_{A}^{0}$, with no huge computational effort. The main idea for the construction of AP-schemes is based on asymptotic arguments and consists in a mathematical reformulation of the singularly perturbed problem $(P)_{A}^{\epsilon}$ into an equivalent problem $(A P)^{\epsilon}$, which is a regular perturbation of the limit problem $(P)_{A}^{0}$. The equivalent reformulation of $(P)_{A}^{\epsilon}$ into $(A P)^{\epsilon}$ is a sort of "reorganization" of the problem into a form which is better suited for the numerical discretization and for the asymptotic limit study $\epsilon \rightarrow 0$. The same numerical scheme is then used for the discretization of $(A P)^{\epsilon}$ as well as for $(P)_{A}^{0}$, which means that this procedure allows for an automatic numerical transition from $(P)_{A}^{\epsilon}$ to $(P)_{A}^{0}$. Considering the singularly-perturbed problem $(P)_{A}^{\epsilon}$, namely (8) in the Anelastic regime, one can think to reformulate it, by introducing the auxiliary variables $\left(p^{\epsilon^{\prime}}, \rho^{\epsilon^{\prime}}\right)$ and solve for every $\epsilon \in[0,1]$ the regular system

$$
(A P)^{\epsilon}\left\{\begin{array}{l}
\partial_{t} \rho^{\epsilon}+\nabla \cdot\left(\rho^{\epsilon} \mathbf{u}^{\epsilon}\right)=0 \\
\rho^{\epsilon}\left[\partial_{t} \mathbf{u}^{\epsilon}+\left(\mathbf{u}^{\epsilon} \cdot \nabla\right) \mathbf{u}^{\epsilon}\right]=-\nabla p^{\epsilon^{\prime}}+\rho^{\epsilon^{\prime}} g \\
-\nabla p^{\epsilon}+\rho^{\epsilon} g=\epsilon^{2}\left[-\nabla p^{\epsilon \prime}+\rho^{\epsilon \prime} g\right] \\
\rho^{\epsilon} T^{\epsilon}\left[\partial_{t} S^{\epsilon}+\left(\mathbf{u}^{\epsilon} \cdot \nabla\right) S^{\epsilon}\right]=\epsilon^{2} \nabla \cdot\left(\rho^{\epsilon} \nabla T^{\epsilon}\right)+\nabla \cdot\left(\rho^{\epsilon} T^{\epsilon} \nabla S^{\epsilon}\right),
\end{array}\right.
$$


completed with the initial conditions (11), boundary conditions as well as the equations of state

$$
\begin{gathered}
p^{\epsilon}=\rho^{\epsilon} T^{\epsilon}, \quad \frac{1}{\gamma} \ln \left(\bar{p}^{\epsilon}\right)-\ln \left(\bar{\rho}^{\epsilon}\right)+1=0, \\
\frac{1}{\gamma} \ln \left(1+\epsilon^{2} \frac{p^{\epsilon^{\prime}}}{\bar{p}^{\epsilon}}\right)-\ln \left(1+\epsilon^{2} \frac{\rho^{\epsilon^{\prime}}}{\bar{\rho}^{\epsilon}}\right)=\epsilon^{2} S^{\epsilon \prime},
\end{gathered}
$$

where we used for simplicity the notation $\bar{p}^{\epsilon}:=p^{\epsilon}-\epsilon^{2} p^{\epsilon \prime}$ as well as $\bar{\rho}^{\epsilon}:=$ $\rho^{\epsilon}-\epsilon^{2} \rho^{\epsilon \prime}$.

To summarize, the asymptotic-preserving approach consists somehow in trying to mimic on the discrete level the asymptotic behaviour of the singularly perturbed problem solutions. It is thus very important to have a full understanding of the solutions behaviour $\left(\rho^{\epsilon}, \mathbf{u}^{\epsilon}, S^{\epsilon}\right)$ of $(A P)^{\epsilon}$. The detailed mathematical and numerical study of this AP-reformulation shall be the aim of a forthcoming paper.

Acknowledgements The author is grateful to Claudia Negulescu (Université Paul Sabatier, Toulouse) for having introduced him to the research field of AP schemes, and for sharing knowledge and ideas that led to this paper.

This work was partially supported by the Italian National Group for Mathematical Physics (GNFM/INdAM).

The author - well aware of his good fortune - would like to express his gratitude and deepest appreciation to Tommaso Ruggeri for being such a supportive, generous and invaluable one-of-a-kind mentor.

\section{References}

1. T. Alazard A minicourse on the low Mach number limit, Discrete Contin. Dyn. Syst. Ser. S 1, 365-404 (2008).

2. P. Degond, F. Deluzet, A. Lozinski, J. Narski, C. Negulescu, Duality based AsymptoticPreserving Method for highly anisotropic diffusion equations, Commun. Math. Sci. 10 (2012), no. 1, 1-31.

3. P. Degond, F. Deluzet, C. Negulescu, An Asymptotic Preserving scheme for strongly anisotropic elliptic problem, SIAM Multiscale Model. Simul. 8 (2010), no. 2, 645-666.

4. P. Degond, A. Lozinski, J. Narski, C. Negulescu, An Asymptotic-Preserving method for highly anisotropic elliptic equations based on a micro-macro decomposition, J. Comput. Phys. 231 (2012), no. 7, 2724-2740.

5. P. Degond, M. Tang, All speed scheme for the low mach number limit of the Isentropic Euler equation, Commun. Comput. Phys. 10, 1-31 (2011).

6. E. Feireisl, A. Novotný, Singular limits in thermodynamics of viscous fluids, Birkhäuser Verlag, Basel, Switzerland, 2009.

7. E. Feireisl, J. Málek, A. Novotný, Y. Straškraba Anelastic approximation as a singular limit of the compressible Navier-Stokes system, Comm. Part. Diff. Eq. 33, 157-176, (2008).

8. P.A. Gilman, G.A. Glatzmaier, Compressible convection in a rotating spherical shell. Part I. Anelastic equations, Astrophys. J. Suppl. Ser. 45, 335-349 (1981).

9. G.A. Glatzmaier, Numerical simulations of stellar convective dynamos. Part I. The model and method, J. Comp. Phys. 55, 461-484 (1984).

10. G.A. Glatzmaier, Numerical simulations of stellar convective dynamos. Part II. Field propagation in the convective zone, Astrophys. J. 291, 300-307 (1985).

11. J. Haack, S. Jin, J.G. Liu, An all-speed asymptotic-preserving method for the isentropic Euler and Navier-Stokes equation, Commun. Comp. Phys. 12, 955-980 (2012). 
12. S. Jin, Efficient Asymptotic-Preserving (AP) Schemes for Some Multiscale Kinetic Equations, SIAM J. Sci. Comp. 21, 441-454 (1999).

13. S. Jin, Asymptotic preserving (AP) schemes for multiscale kinetic and hyperbolic equations: a review, Rivista di Matematica della Universita di Parma 3, 177-216 (2012).

14. C.A. Jones, P. Boronski, A.S. Brun, G.A. Glatzmaier, T. Gastine, M.S. Miesch, J. Wicht, Anelastic convection-driven dynamo benchmarks, Icarus 216, 120-135 (2011).

15. L. Jouve, Modélisation du magnétisme solaire: de son origine interne à ses manifestations en surface, $\mathrm{PhD}$ thesis, Université Paris-Diderot, 2008.

16. S.R. Lantz, Y. Fan, Anelastic magnetohydrodynamic equations for modeling solar and stellar convection zones, Astrophys. J. Suppl. Ser. 121, 247-264 (1999).

17. A. Mentrelli, C. Negulescu, Asymptotic-Preserving scheme for highly anisotropic nonlinear diffusion equations, J. Comput. Phys. 231 (2012), 8229-8245.

18. A. Mentrelli, C. Negulescu, Asymptotic-Preserving scheme for a strongly anisotropic vorticity equation arising in fusion plasma modelling, submitted (2018).

19. M.S. Miesch, Large-Scale Dynamics of the Convection Zone and Tachocline, Living Rev. Solar Phys. 2 (2005)

20. G. I. Ogilvie, Astrophysical Fluid Dynamics, J. Plasma Phys. 82, 205820301 (2016).

21. Y. Ogura, N.A. Phillips, Scale analysis of deep and shallow convection in the athmosphere, J. Atmos. Sci. 19, 173-179 (1962). 